\title{
Plasma concentration of interleukin- 6 was upregulated in cancer cachexia patients and was positively correlated with plasma free fatty acid in female patients
}

\author{
Jun Han ${ }^{1 \dagger}$, Chaocheng Lư ${ }^{1 \dagger}$, Qingyang Meng ${ }^{1}$, Alice Halim², Thong Jia Yean² and Guohao Wu*
}

\begin{abstract}
Background: Cancer cachexia is a clinical manifestation in various advanced cancers that characterized by muscle atrophy and fat loss as its main features; it is frequently associated with systemic inflammatory response. However, the differences in inflammatory response and lipid metabolism of different genders remain unclear. This study explores the difference between cachexic and non-cachexic patients in different genders and cancer types and focus on the plasma inflammation factors levels and lipid metabolism parameters in different genders.

Methods: We first analyzed the general characteristics in 311 cancer patients between cachexic and non-cachexic patients, with an emphasis on expression levels related to inflammatory factors and lipid metabolism parameters. We then further analyzed these characteristics in different genders and cancer types. Lastly, the correlations between plasma interleukin-6 (IL-6) and lipid metabolism parameters in cachexia patients of different genders were analyzed.

Results: Among 311 patients, there were 74 cancer cachexia patients (50 males and 24 females) and 237non-cachexia patients (150 males and 87 females). Body mass index (BMI), TNM stage, plasma concentration of hemoglobin, platelet, lymphocyte count, total protein, albumin, prealbumin, total cholesterol, apolipoprotein $E$ (ApoE), free fatty acid (FFA) and IL- 6 were significantly different between cachexic and non-cachexic patients (all $p<0.05$ ). In addition, these characteristics were different in different cancer types. When compared to male non-cachexic patients, male cachexic patients showed a significant increase in plasma levels of IL-6 and platelet, later TNM stage, with marked decrease in their plasma total protein, albumin, prealbumin, ApoE as well as their lymphocyte counts and hemoglobin levels (all $p<0.05)$. In comparison with female non-cachexic patients, female cachexic patients' IL-6 levels and FFA were significantly elevated with noticeable decrease in their BMl, total cholesterol, ApoE and prealbumin, as well as later TNM stage (all $p<0.05$ ). Correlation analysis revealed that IL-6 levels in female cachexic patients had a significant positive correlation with FFA expression, but this correlation not reflected in male patients.
\end{abstract}

Conclusion: This study demonstrates the different metabolic characteristics of male and female cancer cachexia patients. Future study about cancer cachexia should pay attention to different genders and cancer types.

\footnotetext{
* Correspondence: profwugh@163.com

†un Han and Chaocheng Lu contributed equally to this work.

'Department of General Surgery, Zhongshan Hospital of Fudan University,

180 Fenglin Road, Shanghai 200032, China

Full list of author information is available at the end of the article
}

(c) The Author(s). 2019 Open Access This article is distributed under the terms of the Creative Commons Attribution 4.0 International License (http://creativecommons.org/licenses/by/4.0/), which permits unrestricted use, distribution, and reproduction in any medium, provided you give appropriate credit to the original author(s) and the source, provide a link to the Creative Commons license, and indicate if changes were made. The Creative Commons Public Domain Dedication waiver (http://creativecommons.org/publicdomain/zero/1.0/) applies to the data made available in this article, unless otherwise stated. 


\section{Introduction}

Cancer cachexia occurs in various advanced stage cancers, and approximately $80 \%$ of cancer patients may develop this state. Cancer cachexia is defined as a wasting syndrome characterized by progressive skeletal muscle atrophy and fat loss in cancer patients; it is generally accompanied by systemic inflammatory response, and cannot be fully reversed by conventional nutritional support [1]. Previous studies mainly focused on the muscle atrophy of cancer cachexia, but in recent years, more studies have shown that fat loss also plays an essential role in the occurrence and development of cancer cachexia [2]. The mechanism of fat loss mainly includes increased lipolysis, white-to-brown transdifferentiation of white adipose tissue (WAT browning), and dysfunctional adipocyte synthesis, etc. [3]. Our previous research has shown that patients with cancer cachexia lost fat mass as a result of elevated lipolysis and WAT browning [4]. Previous studies on obesity and metabolic syndromes pointed out the different characteristics of lipid metabolism in opposite genders $[5,6]$. However, whether lipid metabolism differ between different sexes in cancer cachexic is unclear.

Inflammatory response is another important feature of cancer cachexia. Although previous studies showed that plasma interleukin-6 (IL-6), tumor necrosis factor- $\alpha$ (TNF- $\alpha$ ) and other inflammatory factors were significantly elevated in cancer cachexia patients, there were controversies among studies $[7,8]$. In particular, TNF- $\alpha$ expression levels in cancer cachexia patients remained a highly controversial topic. Additionally, studies conducted on animals revealed that IL- 6 expressions were different in cancer cachexic mice of different genders and varied cancer types [9-11]. A deep research of IL-6 expression in opposite genders of cancer cachexic patients is needed.

In this study, we first compared the different clinical characteristics in cancer cachexia patients and noncancer cachexia patients. Subsequently, we the compared different clinical characteristics in patients of opposite genders and different cancer types. Lastly, we analyzed the correlation between lipid metabolism parameters and IL-6 in cancer cachexia patients of different genders.

\section{Methods}

\section{Patients and sample collection}

Patients diagnosed at General Surgery Department of Zhongshan Hospital, Fudan University from January 2018 to June 2019 were included. The inclusion criteria were as follows: (1) Patients were pathologically diagnosed as gastric cancer or colorectal cancer; (2) Patients received radical operation; (3) Patients with complete clinical data. Exclusion criteria includes: (1) Patients received preoperative radiotherapy, chemotherapy or any other pharmacological treatments; (2) Patients accompanied by severe cardiovascular and cerebrovascular diseases, hepatic or renal dysfunction, hyperthyroidism, diabetes or HIV/AIDS; (3) Patients without complete clinical data. $5 \mathrm{ml}$ of venous blood was collected from each patient preoperatively, and was subjected to centrifuge; the plasma obtained was then stored for follow-up tests.

\section{Patient groups}

According to the international consensus of cancer cachexia dated in 2011 [1], this study divided the 311 patients into non-cachexia and cachexia groups respectively based on weight loss and Body Mass Index (BMI). The non-cachexia group is characterized by no weight loss in the past 6 months, or weight loss $<5 \%$, or $\mathrm{BMI}<20$ with weight loss of $<2 \%$; the cachexia group is characterized by weight loss in the past 6 months, with weight loss $>5 \%$ or $\mathrm{BMI}<20$ accompanied by weight loss of $>2 \%$.

\section{Data collection}

From each patient's medical record, height, weight, gender and age were recorded. From their preoperative blood examination results, values of plasma hemoglobin, white blood cell count, platelet, lymphocyte count, total protein, albumin, prealbumin, total cholesterol, triglycerides, apolipoprotein E (ApoE), apolipoprotein B (ApoB), apolipoprotein A (ApoA) and free fatty acid (FFA) were obtained. BMI $=$ Weight $(\mathrm{kg}) /$ height $^{2}\left(\mathrm{~m}^{2}\right)$. We also recorded the TNM stage of each patient according to UICC/AJCC staging system.

\section{Plasma measurement of inflammatory factors}

The plasma concentrations of IL- 6 and TNF- $\alpha$ were determined using a commercialized enzyme-linked immunosorbent assay (ELISA) kit (Thermo Fisher) according to the manufacturer's protocols. Analytical sensitivity of IL- 6 and TNF- $\alpha$ kit is 0.92 and $2.3 \mathrm{pg} / \mathrm{ml}$, with interassay CV for 5.2 and $7.4 \%$ as well as intraassay CV for 3.4 and $6 \%$, respectively.

\section{Statistical analysis}

All statistical analyses were performed using GraphPad Prism 5.0, continuous data were expressed as mean \pm standard error of the mean $\left({ }^{-} \mathrm{x} \pm \mathrm{sem}\right)$, within the context of an independent sample t-test. Categorical data were expressed in frequencies and the $\chi^{2}$ test was adopted. The correlations of serum FFA with IL-6 in different genders of cancer cachexia patients were analyzed using Spearman rank correlation tests. Statistical significance was defined as $p<0.05$. 


\section{Results}

\section{Comparison between cachexia and non-cachexia cancer} patients

Among 311 patients, there were 74 cancer cachexia patients (50 males and 24 females) and 237 non-cachexia patients (150 males and 87 females). Without considering gender, in general, plasma concentrations of IL-6, FFA and platelet of patients in the cancer cachexia group were significantly higher than in the non-cachexia group. In contrast, BMI, plasma concentrations of hemoglobin, lymphocyte count, total protein, albumin, prealbumin, total cholesterol and ApoE of patients in the cancer cachexia group were significantly lower than in non-cachexia group. Furthermore, later TNM stage was more common in cancer cachexia patients (Table 1).

\section{Comparison in different genders between cachexia and non-cachexia cancer patients}

When male cancer cachexia and male non-cachexia patients were compared, IL- 6 and platelet were significantly elevated in the cachexia group, whereas total protein, albumin, prealbumin, ApoE, lymphocyte counts and hemoglobin were significantly reduced in the cachexia group. Similarly, when comparisons were made between female cancer cachexia and female non-cachexia patients, IL-6 and FFA were remarkably higher in the cachexia group, while BMI, total cholesterol, ApoE and prealbumin were apparently lower in the cachexia group. In addition, later TNM stage in cachexic patients was observed in both genders (Table 2). Interestingly, IL-6 levels were significantly elevated in both genders, but FFA was only elevated in female cancer cachexia patients.

\section{Comparison in different cancer types between cachexia and non-cachexia cancer patients}

One hundred seventy-one gastric cancer patients and 140 colorectal cancer patients were involved in this study. Upon further dividing the groups via cancer types (gastric cancer and colorectal cancer), we found that IL6 in gastric cancer cachexia patients was elevated significantly, while their BMI, hemoglobin, lymphocyte count, total protein, albumin, prealbumin, total cholesterol, ApoB and ApoE declined remarkably. In colorectal cancer patients, white blood cell count, platelet, ApoA, FFA, IL- 6 and TNF- $\alpha$ increase significantly, while albumin, prealbumin and ApoE decreased significantly. Interestingly, TNM stages in cachexic patients was later than non-cachexic patients in gastric cancer but not in colorectal cancer (Table 3). The above findings indicated that there were metabolic differences between gastric cancer patients and colorectal cancer patients.

Table 1 Comparison of clinical variables between non-cachexia and cachexia patients

\begin{tabular}{|c|c|c|c|c|}
\hline & Non-cachexia $(n=237)$ & Cachexia $(n=74)$ & $t / x^{2}$ & $p$ \\
\hline Ages (years) & $62.34 \pm 0.73$ & $64.14 \pm 1.19$ & 1.226 & 0.221 \\
\hline Female no. (\%) & $87(36.7 \%)$ & $24(32.4 \%)$ & 0.449 & 0.503 \\
\hline $\mathrm{BMI}\left(\mathrm{kg} / \mathrm{m}^{2}\right)$ & $22.91 \pm 0.19$ & $22.08 \pm 0.38$ & 2.036 & 0.043 \\
\hline $\mathrm{HB}(\mathrm{g} / \mathrm{L})$ & $119.5 \pm 1.68$ & $111.8 \pm 3.02$ & 2.227 & 0.027 \\
\hline WBC $(10 \wedge 9 / L)$ & $5.72 \pm 0.11$ & $6.07 \pm 0.25$ & 1.422 & 0.156 \\
\hline PLT $(10 \wedge 9 / L)$ & $237.9 \pm 5.15$ & $262.9 \pm 11.75$ & 2.211 & 0.028 \\
\hline $\mathrm{LY}(10 \wedge 9 / L)$ & $1.64 \pm 0.04$ & $1.40 \pm 0.05$ & 3.168 & 0.002 \\
\hline TP $(g / L)$ & $65.36 \pm 0.41$ & $63.64 \pm 0.78$ & 2.016 & 0.045 \\
\hline ALB $(\mathrm{g} / \mathrm{L})$ & $40.88 \pm 0.32$ & $39.15 \pm 0.56$ & 2.675 & 0.008 \\
\hline PA (g/L) & $0.214 \pm 0.004$ & $0.189 \pm 0.006$ & 3.525 & $<0.001$ \\
\hline $\mathrm{TC}(\mathrm{mmol} / \mathrm{L})$ & $4.19 \pm 0.07$ & $3.91 \pm 0.09$ & 2.149 & 0.033 \\
\hline $\mathrm{TG}(\mathrm{mmol} / \mathrm{L})$ & $1.32 \pm 0.04$ & $1.17 \pm 0.06$ & 1.902 & 0.058 \\
\hline ApoA (g/l) & $176.8 \pm 12.62$ & $220.1 \pm 23.61$ & 1.677 & 0.095 \\
\hline ApoB $(g / l)$ & $0.873 \pm 0.017$ & $0.852 \pm 0.029$ & 0.604 & 0.547 \\
\hline ApoE $(g / l)$ & $41.55 \pm 0.82$ & $36.78 \pm 1.36$ & 2.919 & 0.004 \\
\hline $\mathrm{FFA}(\mathrm{pg} / \mathrm{ml})$ & $0.42 \pm 0.02$ & $0.48 \pm 0.03$ & 2.022 & 0.044 \\
\hline IL-6 (pg/ml) & $4.88 \pm 0.28$ & $8.16 \pm 0.98$ & 4.429 & $<0.001$ \\
\hline TNF-a (pg/ml) & $7.45 \pm 0.23$ & $8.09 \pm 0.37$ & 1.398 & 0.164 \\
\hline$(I+I I)$ TNM stage no. (\%) & $125(52.7 \%)$ & $24(32.4 \%)$ & 9.321 & 0.002 \\
\hline
\end{tabular}

BMI Body mass index, HB Hemoglobin, WBC White blood cell count, PLT Platelet, $L Y$ Lymphocyte count, TP Total protein, ALB Albumin, PA Prealbumin, TC Total cholesterol, TG Tri-glyceride, ApoA Apolipoprotein A, ApoB Apolipoprotein B, ApoE Apolipoprotein E, FFA Free fatty acid 
Table 2 Comparison in different genders between cachexia and non-cachexia cancer patients

\begin{tabular}{|c|c|c|c|c|c|c|}
\hline & \multicolumn{3}{|l|}{ male } & \multicolumn{3}{|l|}{ female } \\
\hline & Non-cachexia $(n=150)$ & Cachexia $(n=50)$ & $p$ & Non-cachexia $(n=87)$ & Cachexia $(n=24)$ & $p$ \\
\hline Ages (years) & $62.97 \pm 0.85$ & $65.32 \pm 1.24$ & 0.155 & $61.24 \pm 1.33$ & $61.67 \pm 2.59$ & 0.883 \\
\hline $\mathrm{BMI}\left(\mathrm{kg} / \mathrm{m}^{2}\right)$ & $22.82 \pm 0.24$ & $22.26 \pm 0.47$ & 0.266 & $23.11 \pm 0.33$ & $21.65 \pm 0.67$ & 0.047 \\
\hline $\mathrm{HB}(\mathrm{g} / \mathrm{L})$ & $125.9 \pm 1.95$ & $115.6 \pm 3.92$ & 0.012 & $108.3 \pm 2.68$ & $103.8 \pm 3.99$ & 0.416 \\
\hline WBC (10^9/L) & $5.83 \pm 0.15$ & $6.13 \pm 0.31$ & 0.335 & $5.51 \pm 0.18$ & $5.92 \pm 0.40$ & 0.314 \\
\hline $\mathrm{PLT}(10 \wedge 9 / \mathrm{L})$ & $232.2 \pm 5.86$ & $259.1 \pm 14.07$ & 0.039 & $241.9 \pm 9.46$ & $271.1 \pm 21.73$ & 0.174 \\
\hline $\operatorname{LY}(10 \wedge 9 / L)$ & $1.61 \pm 0.04$ & $1.41 \pm 0.08$ & 0.023 & $1.66 \pm 0.06$ & $1.55 \pm 0.09$ & 0.376 \\
\hline $\mathrm{TP}(\mathrm{g} / \mathrm{L})$ & $65.18 \pm 0.43$ & $63.15 \pm 0.88$ & 0.026 & $66.16 \pm 0.77$ & $65.52 \pm 1.72$ & 0.715 \\
\hline ALB $(g / L)$ & $40.93 \pm 0.36$ & $39.3 \pm 0.66$ & 0.027 & $40.78 \pm 0.60$ & $38.81 \pm 1.08$ & 0.128 \\
\hline $\mathrm{PA}(\mathrm{g} / \mathrm{L})$ & $0.219 \pm 0.005$ & $0.186 \pm 0.008$ & $<0.001$ & $0.199 \pm 0.006$ & $0.173 \pm 0.011$ & 0.039 \\
\hline $\mathrm{TC}(\mathrm{mmol} / \mathrm{L})$ & $3.97 \pm 0.07$ & $3.84 \pm 0.12$ & 0.379 & $4.53 \pm 0.11$ & $4.05 \pm 0.17$ & 0.043 \\
\hline $\mathrm{TG}(\mathrm{mmol} / \mathrm{L})$ & $1.28 \pm 0.05$ & $1.18 \pm 0.06$ & 0.235 & $1.35 \pm 0.08$ & $1.24 \pm 0.14$ & 0.501 \\
\hline ApoA (g/l) & $155.2 \pm 13.81$ & $189.5 \pm 23.05$ & 0.206 & $187.3 \pm 19.86$ & $281.6 \pm 60.36$ & 0.057 \\
\hline ApoB $(g / l)$ & $0.84 \pm 0.02$ & $0.84 \pm 0.04$ & 0.905 & $0.94 \pm 0.03$ & $0.87 \pm 0.05$ & 0.312 \\
\hline ApoE $(g / l)$ & $39.37 \pm 0.98$ & $34.64 \pm 1.49$ & 0.013 & $46.85 \pm 1.65$ & $39.16 \pm 2.28$ & 0.028 \\
\hline FFA $(p g / m l)$ & $0.39 \pm 0.02$ & $0.43 \pm 0.03$ & 0.332 & $0.43 \pm 0.03$ & $0.56 \pm 0.06$ & 0.020 \\
\hline IL-6 (pg/ml) & $5.23 \pm 0.37$ & $6.95 \pm 0.84$ & 0.037 & $4.28 \pm 0.40$ & $7.55 \pm 1.25$ & 0.001 \\
\hline TNF-a (pg/ml) & $7.547 \pm 0.283$ & $7.654 \pm 0.424$ & 0.849 & $9.290 \pm 0.875$ & $8.09 \pm 0.3719$ & 0.179 \\
\hline (I + II) TNM stage no. (\%) & $76(50.7 \%)$ & $16(32.0 \%)$ & 0.022 & $49(56.3 \%)$ & $8(33.3 \%)$ & 0.046 \\
\hline
\end{tabular}

BMI Body mass index, HB Hemoglobin, WBC White blood cell count, PLT Platelet, $L Y$ Lymphocyte count, TP Total protein, ALB Albumin, PA Prealbumin, TC Total cholesterol, TG Tri-glyceride, ApoA Apolipoprotein A, ApoB Apolipoprotein B, ApoE Apolipoprotein E, FFA Free fatty acid

Table 3 Comparison in different cancer types between cachexia and non-cachexia cancer patients

\begin{tabular}{|c|c|c|c|c|c|c|}
\hline & Gastric cancer & & & Colorectal cancer & & \\
\hline & Non-cachexia $(n=126)$ & Cachexia $(n=45)$ & $p$ & Non-cachexia $(n=111)$ & Cachexia $(n=29)$ & $p$ \\
\hline Ages (years) & $63.27 \pm 0.92$ & $64.34 \pm 1.33$ & 0.352 & $61.84 \pm 1.63$ & $62.21 \pm 2.09$ & 0.423 \\
\hline $\mathrm{BMI}\left(\mathrm{kg} / \mathrm{m}^{2}\right)$ & $23.23 \pm 0.27$ & $21.76 \pm 0.47$ & 0.008 & $22.71 \pm 0.31$ & $22.74 \pm 0.70$ & 0.965 \\
\hline $\mathrm{HB}(\mathrm{g} / \mathrm{L})$ & $121.2 \pm 2.27$ & $111.6 \pm 4.36$ & 0.038 & $119.2 \pm 2.64$ & $111.4 \pm 4.38$ & 0.147 \\
\hline WBC $(10 \wedge 9 / L)$ & $5.62 \pm 0.15$ & $5.51 \pm 0.33$ & 0.711 & $5.93 \pm 0.17$ & $7.04 \pm 0.34$ & 0.002 \\
\hline PLT $(10 \wedge 9 / L)$ & $238.2 \pm 7.39$ & $247.3 \pm 15.07$ & 0.561 & $241.2 \pm 7.83$ & $281.3 \pm 18.35$ & 0.023 \\
\hline $\mathrm{LY}(10 \wedge 9 / L)$ & $1.66 \pm 0.05$ & $1.41 \pm 0.09$ & 0.012 & $1.58 \pm 0.06$ & $1.55 \pm 0.08$ & 0.793 \\
\hline TP $(g / L)$ & $65.31 \pm 0.59$ & $61.81 \pm 1.03$ & 0.004 & $65.67 \pm 0.52$ & $66.96 \pm 1.15$ & 0.252 \\
\hline ALB $(\mathrm{g} / \mathrm{L})$ & $40.53 \pm 0.44$ & $38.41 \pm 0.72$ & 0.019 & $41.23 \pm 0.46$ & $39.10 \pm 0.78$ & 0.017 \\
\hline $\mathrm{PA}(\mathrm{g} / \mathrm{L})$ & $0.219 \pm 0.005$ & $0.198 \pm 0.008$ & 0.032 & $0.208 \pm 0.006$ & $0.178 \pm 0.009$ & 0.008 \\
\hline $\mathrm{TC}(\mathrm{mmol} / \mathrm{L})$ & $4.18 \pm 0.09$ & $3.74 \pm 0.14$ & 0.012 & $4.177 \pm 0.10$ & $4.273 \pm 0.14$ & 0.621 \\
\hline $\mathrm{TG}(\mathrm{mmol} / \mathrm{L})$ & $1.35 \pm 0.06$ & $1.19 \pm 0.11$ & 0.19 & $1.23 \pm 0.06$ & $1.32 \pm 0.09$ & 0.404 \\
\hline ApoA (g/l) & $190.5 \pm 18.68$ & $188.2 \pm 27.12$ & 0.948 & $157.1 \pm 16.62$ & $277.2 \pm 42.97$ & 0.002 \\
\hline ApoB $(g / l)$ & $0.88 \pm 0.02$ & $0.77 \pm 0.04$ & 0.019 & $0.87 \pm 0.03$ & $0.97 \pm 0.04$ & 0.090 \\
\hline ApoE $(g / l)$ & $40.12 \pm 0.99$ & $35.74 \pm 1.80$ & 0.032 & $43.26 \pm 1.49$ & $37.32 \pm 1.88$ & 0.037 \\
\hline $\mathrm{FFA}(\mathrm{pg} / \mathrm{ml})$ & $0.41 \pm 0.02$ & $0.45 \pm 0.04$ & 0.254 & $0.425 \pm 0.024$ & $0.53 \pm 0.05$ & 0.041 \\
\hline IL-6 (pg/ml) & $4.60 \pm 0.37$ & $6.82 \pm 1.02$ & 0.014 & $5.04 \pm 0.42$ & $7.38 \pm 0.95$ & 0.013 \\
\hline TNF-a (pg/ml) & $7.94 \pm 0.42$ & $8.05 \pm 0.63$ & 0.894 & $7.32 \pm 0.382$ & $9.01 \pm 0.553$ & 0.037 \\
\hline (I + II) TNM stage no. (\%) & $70(55.6 \%)$ & 17 (35.6\%) & 0.023 & $51(46.9 \%)$ & $14(41.4 \%)$ & 0.660 \\
\hline
\end{tabular}




\section{Comparison in cancer cachexia between male and female patients}

Direct comparisons were made between male and female cancer cachexia patients, which revealed significant differences in FFA and ApoE in opposite genders (Table 4). No apparent difference was detected in other characteristics.

\section{Correlation analyses between plasma IL- 6 and lipid metabolism parameters in cachexia patients of different genders}

We focused on analyzing the correlation of IL-6 and lipid metabolism parameters, and discovered a positive correlation between plasma IL-6 and FFA in female cancer cachexia patients, but no significant correlation were observed in male patients (Table 5).

\section{Discussion}

Cancer cachexia generally accompanied by systemic inflammatory response and fat loss. Previous studies mainly divided cachexia patients by degree of weight loss, but seldom consider the differences between genders. This study, through analyzing 311 gastric cancer and colorectal cancer patients, found that the inflammatory factor, IL-6, was significantly elevated in male and female patients, but TNF$\alpha$, another common inflammatory factor concerning

Table 4 Comparison in cancer cachexia between male and female patients

\begin{tabular}{|c|c|c|c|}
\hline & Cachexia & & \\
\hline & Male $(n=50)$ & Female $(n=24)$ & $p$ \\
\hline Ages (years) & $65.32 \pm 1.24$ & $62.83 \pm 2.42$ & 0.313 \\
\hline BMI $\left(\mathrm{kg} / \mathrm{m}^{2}\right)$ & $22.26 \pm 0.47$ & $21.65 \pm 0.67$ & 0.461 \\
\hline $\mathrm{HB}(\mathrm{g} / \mathrm{L})$ & $115.6 \pm 3.92$ & $103.8 \pm 3.99$ & 0.067 \\
\hline WBC (10^9/L) & $6.13 \pm 0.31$ & $5.92 \pm 0.40$ & 0.697 \\
\hline PLT (10^9/L) & $259.1 \pm 14.07$ & $271.1 \pm 21.73$ & 0.637 \\
\hline LY $(10 \wedge 9 / L)$ & $1.40 \pm 0.08$ & $1.55 \pm 0.09$ & 0.295 \\
\hline $\mathrm{TP}(\mathrm{g} / \mathrm{L})$ & $63.15 \pm 0.88$ & $65.52 \pm 1.72$ & 0.179 \\
\hline ALB $(g / L)$ & $39.31 \pm 0.66$ & $38.81 \pm 1.08$ & 0.686 \\
\hline $\mathrm{PA}(\mathrm{g} / \mathrm{L})$ & $0.186 \pm 0.008$ & $0.178 \pm 0.011$ & 0.557 \\
\hline $\mathrm{TC}(\mathrm{mmol} / \mathrm{L})$ & $3.84 \pm 0.12$ & $4.13 \pm 0.18$ & 0.191 \\
\hline $\mathrm{TG}(\mathrm{mmol} / \mathrm{L})$ & $1.216 \pm 0.07$ & $1.241 \pm 0.14$ & 0.858 \\
\hline ApoA $(g / l)$ & $202.1 \pm 25.81$ & $246.2 \pm 51.42$ & 0.395 \\
\hline ApoB $(g / l)$ & $0.84 \pm 0.04$ & $0.87 \pm 0.05$ & 0.648 \\
\hline ApoE $(g / l)$ & $34.64 \pm 1.49$ & $41.38 \pm 2.57$ & 0.019 \\
\hline FFA $(p g / m l)$ & $0.43 \pm 0.03$ & $0.56 \pm 0.06$ & 0.029 \\
\hline IL-6 (pg/ml) & $7.47 \pm 1.05$ & $7.55 \pm 1.25$ & 0.961 \\
\hline TNF-a $(\mathrm{pg} / \mathrm{ml})$ & $7.65 \pm 0.42$ & $8.74 \pm 0.71$ & 0.171 \\
\hline (I + II) TNM stage no. (\%) & $16(32.0 \%)$ & $8(33.3 \%)$ & 0.909 \\
\hline
\end{tabular}

$B M I$ Body mass index, $H B$ Hemoglobin, WBC White blood cell count, $P L T$ Platelet, $L Y$ Lymphocyte count, TP Total protein, ALB Albumin, PA Prealbumin, TC Total cholesterol, TG Tri-glyceride, ApoA Apolipoprotein A, ApoB Apolipoprotein B, ApoE Apolipoprotein E, FFA Free fatty acid
Table 5 Correlation analyses between plasma IL-6 and lipid metabolism parameters in cachexia patients of different genders

\begin{tabular}{llllll}
\hline & \multicolumn{2}{l}{ Male } & & & Female \\
\cline { 2 - 3 } & $r$ & & & & \\
\hline TC & -0.19 & 0.247 & & 0.02 & 0.933 \\
TG & -0.19 & 0.248 & & -0.09 & 0.698 \\
ApoA & 0.08 & 0.632 & 0.06 & 0.805 \\
ApoB & -0.10 & 0.543 & & 0.35 & 0.157 \\
ApoE & -0.23 & 0.168 & & -0.23 & 0.357 \\
FFA & -0.19 & 0.24 & 0.47 & 0.037 \\
\hline
\end{tabular}

TC Total cholesterol, TG Tri-glyceride, ApoA Apolipoprotein A, ApoB Apolipoprotein B, ApoE Apolipoprotein E, FFA Free fatty acid

cachexia, had no apparent change in both male and female patients, indicating that serum IL-6 might play a more important role during the progress of cachexia. In addition, FFA was significantly elevated in female cancer cachexia patients only, which strongly suggests that male and female cancer cachexia patients have metabolic differences.

In this study, we first compared the differences in clinical data between cancer cachexia and non-cachexia patients, regardless of gender; albumin, prealbumin, lymphocyte count and other conventional nutritional markers decreased in cachexia patients. Incidentally, we found that blood platelet levels in cancer cachexia patients were significantly elevated, which might be associated with the "first responder" properties of platelet during chronic inflammation, cancer progression and metastasis [12]. However, the detailed mechanism remains to be determined. Upon further investigating according to genders, we found distinct differences of many markers in male and female cachexia patients compared with non-cachexia patients. These findings suggested that different genders should be separately analyzed in future investigation regarding the cancer cachexia.

Previous studies mainly emphasized on muscle atrophy and its mechanism during cancer cachexia. Our previous study also revealed that inflammatory factors promoted muscle loss in cancer cachexia patients [13]. However, recent studies have revealed that fat loss might play a crucial role in the progress of cancer cachexia [7]. In this study, we incidentally found that plasma concentration of ApoE significantly decreased in cancer cachexia patients, which was the first report as far as we known. ApoE a component of chylomicrons (CM), very low-density lipoproteins (VLDL), as well as the remnants and $\beta$-VLDL. The concentration of ApoE showed positive correlation with plasma triglyceride level [14]. ApoE genotype has the most profound genetic risk on late onset Alzheimer's disease and also affects processes in normal brains $[15,16]$. In Alzheimer's disease, ApoE interactions with molecules important for lipid efflux and lipid endocytosis underlie effects of ApoE genotype on 
neuroinflammation and lipoprotein composition [15]. The role of ApoE in cancer cells is unclear and limited studies have addressed it. ApoE has been found to be related to lung cancer and considered to be a useful marker for assessing NSCLC patients with lymph node metastasis [17]. ApoE was also recognized as new pancreatic cancer biomarker but had no association with risk of prostate cancer $[18,19]$. The role of ApoE in cancer cells is unclear except that ApoE promoting cancer cell proliferation and migration [20, 21]. In our study, plasma concentration of ApoE was downregulated in cancer cachexia patients compared to non-cancer cachexia patients regardless of genders and cancer types, indicating its important role during the progress of cancer cachexia. However, further study is needed to investigate the role of ApoE in cancer cachexia.

FFA is the primary fuel for heart and skeletal muscles and are precursors of hormones and non-hormonal signalling molecules. FFA plays important physiological roles in skeletal muscle, heart, liver and pancreas. However, chronically elevated plasma FFA appear to have pathophysiological consequences [22]. Elevated FFA was linked with the onset of insulin resistance and associated with type-2 diabetes, by inhibiting the tyrosine phosphorylation of IRS-1 (insulin receptor substrate-1) and reducing IRS-1-ssociated PI3K (phosphatidyl-inositol 3kinase) activity which is responsible for transducing downstream insulin signals $[23,24]$. In this study, we screened out the influences of diabetes, hyperthyroidism and other related diseases to investigate the association of FFA and cancer cachexia induced lipolysis. When we studied cancer cachexia patients according to gender, we discovered that FFA increased significantly in female patients only, while male patients demonstrated an elevating trend. Studies have reported greater serum FFA concentrations in estrogen-deficient women compared with postmenopausal women receiving estrogen treatment, suggesting that estrogen treatment has beneficial effects in postmenopausal women by preventing the accelerated delivery of FFA into the circulation [25]. Estrogen may attenuate lipolysis occurring in adipose tissue through the up-regulation of $\alpha 2$-adrenergic receptors, resulting in decreased mobilization of FFA from adipose tissue [26]. Estrogen also regulated the FFA release by regulating the hormone sensitive lipase (HSL) and lipoprotein lipase (LPL) [27]. In our study, 5 out of $24 \mathrm{fe-}$ male (20.8\%) cachexic patients and 15 out of 87 female (17.2\%) non-cachexic patients were less than 50 years old, indicating the estrogen reduction might have a similar effect on the FFA in both groups. Therefore, the elevated concentration of FFA in female cachexic patients might be attribute to the abnormal metabolism of fat tissues and further study should be conducted to analyze the specific mechanisms.
Studies have shown that inflammatory factors (especially IL- 6 and TNF- $\alpha$ ) play an important role in the occurrence and development of cancer cachexia [8]. In animal cachexia models, both plasma IL- 6 and TNF- $\alpha$ were significantly increased [28]. However, the serum results of cancer cachexia patients were controversial [29], and that inhibiting TNF- $\alpha$ in cancer cachexia patients with weight loss did not halt the process of muscle loss in patients [30]. TNF- $\alpha$ is one inflammatory mediator that has been implicated in carcinogenesis, due to its participation in chronic inflammatory diseases [31]. There is controversy; however, regarding the role of TNF- $\alpha$ in cancer, the pro- or antitumoral TNF- $\alpha$ response with in the tumor microenvironment depends not only on local concentration but also on its expression site in the tumor [32]. Studies have reported the involvement of TNF- $\alpha$ in lipolysis by decreasing LPL activities [33]. TNF- $\alpha$ was also a potent inhibitor of lipogenesis and suppressed the expression of gene responsible for various nuclear transcription factors that were required for differentiation and function of adipocyte [33]. These findings indicate the role of TNF- $\alpha$ in adipose tissue loss and in the development of cancer cachexia [34]. Our study showed that no apparent changes of plasma TNF- $\alpha$ in cancer cachexia patients were observed in both genders. However, TNF- $\alpha$ was increased in colorectal cancer cachexia patient, indicating that TNF- $\alpha$ might play a role in the development of cancer cachexia in colorectal cancer cachexia but not in gastric cancer cachexia.

Conversely, serum IL-6 level was significantly elevated in both male and female cancer cachexia patients, which strongly implied that IL-6 plays an important role in the progress of cancer cachexia. Previous studies implicated IL-6-STAT3 in fat wasting and the acute phase response in cancer cachexia [35]. We found a significant positive correlation between IL- 6 and FFA levels in female cancer cachexia patients but no correlation was observed in male cancer cachexia patients. This finding suggests that IL-6 may be a primary factor in promoting lipolysis in female patients with cancer cachexia, resulting in elevated FFA.

\section{Limitations}

The main limitation of this study was a relatively limited sample size. Only 24 females cancer cachexia patients were included and when we conducted subgroup analysis might cause the potential bias. We will include more patients to validate the results in further studies. Furthermore, only two cancer types (gastric cancer and colorectal cancer) are investigated in this study and whether other cancer types such as pancreatic cancer showed the similar characteristics is not convinced. We 
will include more patients with different cancer types in further studies.

\section{Conclusion}

In conclusion, by analyzing the differences in male and female cancer cachexia patients, we discovered different metabolic characteristics between each gender. In addition, patients with different cancer types also showed different metabolic characteristics. Plasma IL-6 was significantly elevated in both genders, but FFA was only elevated in females and positively associated with IL-6. Our findings from this research serve as a reminder in the future to study and treat cancer cachexia patients with respect to their gender and cancer types.

\section{Abbreviations}

ALB: Albumin; ApoA: Apolipoprotein A; ApoB: Apolipoprotein B ApoE: Apolipoprotein E; BMI: Body mass index; FFA: Free fatty acid; HB: Hemoglobin; LY: Lymphocyte count; PA: Prealbumin; PLT: Platelet; TC: Total cholesterol; TG: Tri-glyceride; TP: Total protein; WBC: White blood cell count

\section{Acknowledgements}

We thank all patients for their participation.

\section{Authors' contributions}

$\mathrm{JH}$ and $\mathrm{GW}$ conceived and designed the experiments. $\mathrm{JH}, \mathrm{CL}$ and $\mathrm{QM}$ collected the data. $\mathrm{JH}$ and $\mathrm{CL}$ analysed and interpreted the data. $\mathrm{JH}, \mathrm{AM}$ and

TJ wrote the paper. All authors reviewed and approved the paper.

\section{Funding}

This research was supported by grants from the Youth of National Natural Science Foundation of China (81803091).

\section{Availability of data and materials}

The datasets during and/or analyzed during the current study available from the corresponding author on reasonable request.

\section{Ethics approval and consent to participate}

This study was approved by the Ethics Committee of Zhongshan Hospital, Fudan University (B2013-106R). All study participants were included in the study after giving written informed consent.

\section{Consent for publication}

Not applicable.

\section{Competing interests}

The authors declare no conflicts of interest.

\section{Author details}

'Department of General Surgery, Zhongshan Hospital of Fudan University, 180 Fenglin Road, Shanghai 200032, China. ${ }^{2}$ Shanghai Medical College, Fudan University, 138 Yixueyuan Road, Shanghai 200032, China.

Received: 17 September 2019 Accepted: 6 November 2019 Published online: 15 November 2019

\section{References}

1. Fearon K, Strasser F, Anker SD, et al. Definition and classification of cancer cachexia: an international consensus. Lancet Oncol. 2011;12:489-95.

2. Vaitkus JA, Celi FS. The role of adipose tissue in cancer-associated cachexia Exp Biol Med (Maywood). 2017:242:473-81.

3. Fearon K, Arends J, Baracos V. Understanding the mechanisms and treatment options in cancer cachexia. Nat Rev Clin Oncol. 2013;10:90-9.

4. Han J, Meng Q, Shen L, et al. Interleukin-6 induces fat loss in cancer cachexia by promoting white adipose tissue lipolysis and browning. Lipids Health Dis. 2018;17:14.
5. de Mutsert R, Gast K, Widya R, et al. Associations of abdominal subcutaneous and visceral fat with insulin resistance and secretion differ between men and women: the Netherlands epidemiology of obesity study. Metab Syndr Relat Disord. 2018:16:54-63.

6. Lagarrigue A, Ajana S, Capuron L, et al. Obesity in French inmates: gender differences and relationship with mood, Eating Behavior and Physical Activity. PLoS One. 2017;12:e0170413.

7. Tsoli M, Swarbrick MM, Robertson GR. Lipolytic and thermogenic depletion of adipose tissue in cancer cachexia. Semin Cell Dev Biol. 2016;54:68-81.

8. Loumaye A, Thissen JP. Biomarkers of cancer cachexia. Clin Biochem. 2017; 50:1281-8.

9. Hetzler KL, Hardee JP, Puppa MJ, et al. Sex differences in the relationship of IL-6 signaling to cancer cachexia progression. Biochim Biophys Acta. 1852; 2015:816-25.

10. Caetano MS, Hassane M, Van HT, et al. Sex specific function of epithelial STAT3 signaling in pathogenesis of K-ras mutant lung cancer. Nat Commun. 2018;9:4589.

11. Ramsey ML, Talbert E, Ahn D, et al. Circulating interleukin- 6 is associated with disease progression, but not cachexia in pancreatic cancer. Pancreatology. 2019;19:80-7.

12. Menter DG, Kopetz S, Hawk E, et al. Platelet "first responders" in wound response, cancer, and metastasis. Cancer Metastasis Rev. 2017;36:199-213.

13. Yuan L, Han J, Meng Q, et al. Muscle-specific E3 ubiquitin ligases are involved in muscle atrophy of cancer cachexia: an in vitro and in vivo study. Oncol Rep. 2015;33:2261-8.

14. Tao QQ, Chen Y, Liu ZJ, et al. Associations between apolipoprotein E genotypes and serum levels of glucose, cholesterol, and triglycerides in a cognitively normal aging Han Chinese population. Clin Interv Aging. 2014;9: 1063-7.

15. Rebeck GW. The role of APOE on lipid homeostasis and inflammation in normal brains. J Lipid Res. 2017;58:1493-9.

16. Lane-Donovan C, Herz J. ApoE, ApoE receptors, and the synapse in Alzheimer's disease. Trends Endocrinol Metab. 2017;28:273-84.

17. An HJ, Koh HM, Song DH. Apolipoprotein E is a predictive marker for assessing non-small cell lung cancer patients with lymph node metastasis. Pathol Res Pract. 2019:215:152607.

18. Liu H, Shui IM, Platz EA, et al. No association of ApoE genotype with risk of prostate Cancer: a nested case-control study. Cancer Epidemiol Biomark Prev. 2015;24:1632-4.

19. Liu X, Zheng W, Wang W, et al. A new panel of pancreatic cancer biomarkers discovered using a mass spectrometry-based pipeline. $\mathrm{Br} \mathrm{J}$ Cancer. 2017;117:1846-54.

20. Su WP, Chen YT, Lai WW, et al. Apolipoprotein E expression promotes lung adenocarcinoma proliferation and migration and as a potential survival marker in lung cancer. Lung Cancer. 2011;71:28-33.

21. Chen YC, Pohl G, Wang TL, et al. Apolipoprotein $E$ is required for cell proliferation and survival in ovarian cancer. Cancer Res. 2005:65:331-7.

22. Ghosh A, Gao L, Thakur A, et al. Role of free fatty acids in endothelial dysfunction. J Biomed Sci. 2017:24:50.

23. Sobczak A IS, Blindauer C A, Stewart A J. Changes in Plasma Free Fatty Acids Associated with Type-2 Diabetes. Nutrients. 2019;11:2022.

24. Sears $B$, Perry $M$. The role of fatty acids in insulin resistance. Lipids Health Dis. 2015;14:121.

25. Kim JH, Cho HT, Kim YJ. The role of estrogen in adipose tissue metabolism: insights into glucose homeostasis regulation. Endocr J. 2014;61:1055-67.

26. Pedersen SB, Kristensen K, Hermann PA, et al. Estrogen controls lipolysis by up-regulating alpha2A-adrenergic receptors directly in human adipose tissue through the estrogen receptor alpha. Implications for the female fat distribution. J Clin Endocrinol Metab. 2004;89:1869-78.

27. Homma $H$, Kurachi $H$, Nishio $Y$, et al. Estrogen suppresses transcription of lipoprotein lipase gene. Existence of a unique estrogen response element on the lipoprotein lipase promoter. J Biol Chem. 2000;275: 11404-11.

28. Baltgalvis KA, Berger FG, Peña MM, et al. Muscle wasting and interleukin-6induced atrogin-I expression in the cachectic Apc ( $\mathrm{min} /+$ ) mouse. Pflugers Arch. 2009:457:989-1001.

29. Grim-Stieger M, Keilani M, Mader RM, et al. Serum levels of tumour necrosis factor-alpha and interleukin- 6 and their correlation with body mass index, weight loss, appetite and survival rate--preliminary data of Viennese outpatients with metastatic cancer during palliative chemotherapy. Eur J Cancer Care (Engl). 2008;17:454-62. 
30. Jatoi A, Ritter HL, Dueck A, et al. A placebo-controlled, double-blind trial of infliximab for cancer-associated weight loss in elderly and/or poor performance non-small cell lung cancer patients (N01C9). Lung Cancer. 2010;68:234-9.

31. Popa C, Netea MG, van Riel PL, et al. The role of TNF-alpha in chronic inflammatory conditions, intermediary metabolism, and cardiovascular risk. J Lipid Res. 2007;48:751-62.

32. Landskron $\mathrm{G}$, De la Fuente $\mathrm{M}$, Thuwajit $\mathrm{P}$, et al. Chronic inflammation and cytokines in the tumor microenvironment. J Immunol Res. 2014;2014: 149185.

33. Sethi JK, Hotamisligil GS. The role of TNF alpha in adipocyte metabolism. Semin Cell Dev Biol. 1999;10:19-29.

34. Patel HJ, Patel BM. TNF-a and cancer cachexia: molecular insights and clinical implications. Life Sci. 2017;170:56-63.

35. Zimmers TA, Fishel ML, Bonetto A. STAT3 in the systemic inflammation of cancer cachexia. Semin Cell Dev Biol. 2016;54:28-41.

\section{Publisher's Note}

Springer Nature remains neutral with regard to jurisdictional claims in published maps and institutional affiliations.

Ready to submit your research? Choose BMC and benefit from:

- fast, convenient online submission

- thorough peer review by experienced researchers in your field

- rapid publication on acceptance

- support for research data, including large and complex data types

- gold Open Access which fosters wider collaboration and increased citations

- maximum visibility for your research: over $100 \mathrm{M}$ website views per year

At BMC, research is always in progress.

Learn more biomedcentral.com/submissions 